\title{
A Survey of Sketch Based Modeling Systems
}

\author{
Ismail Khalid Kazmi, Lihua You, Jian Jun Zhang \\ National Center for Computer Animation \\ Bournemouth University \\ Bournemouth, UK \\ email: ikazmi@bournemouth.ac.uk, lyou@bournemouth.ac.uk, jzhang@bournemouth.ac.uk
}

\begin{abstract}
D modeling is an intricate and laboriously process which requires considerable investment of time to master. Researchers have long pondered on creating systems that would automate the steps followed by skilled $3 D$ artists to generate detailed and realistic $3 D$ models. Sketch Based Modeling (SBM) is a very broad area within the field of Computer Science spanning several sub-fields such as Computational Geometry, 3D Shape Retrieval, Computer Vision, Computer Graphics, and Object Oriented Programming to name a few. The interpretation of sketches to assist artists to create $3 D$ models serves as the main idea behind efficient SBM systems. In this paper we have reviewed and analyzed recent and salient state-of-art techniques in Sketch Based Modeling. We have provided a classification of these techniques and pointed out their advantages and disadvantages.
\end{abstract}

\section{Introduction}

3D modeling is an intricate and laboriously process which requires considerable investment of time to master. Most skilled 3D artists generally possess years of modeling experience and thorough understanding of drawing skills (human anatomy, still life, environments, machinery, vehicles etc) which allow them to create 3D models quickly and accurately. Researchers have long pondered on creating systems that would automate the steps followed by skilled 3D artists to generate detailed and realistic 3D models. Professional modeling packages such as Maya [Maya], ZBrush [Zbrush] and Mudbox [Mudbox] are loaded with tools that make modeling easy, fun and swift for artists of intermediate to expert levels, however these packages have steep learning curves for beginners, purely work on 3D input data and does not support the interpretation of sketched lines and curves into 3D geometry thus limiting the scope of adding details to $3 \mathrm{D}$ models.

Sketch Based Modeling (SBM) is a very broad area within the field of Computer Science spanning several sub-fields such as Computational Geometry, 3D Shape Retrieval, Computer Vision, Computer Graphics, and Object Oriented Programming to name a few. The interpretation of sketches to assist artists to create $3 \mathrm{D}$ models serves as the main idea behind efficient SBM systems. The research in SBM can be traced back to as early as the mid 1980's. Among the early works, John Canny published his seminal work in edge detection famously known as Canny Edge Detection algorithm [Canny 1986]. This algorithm is used to extract feature curves from 2D images including silhouette and local feature curves. Researchers have explored many avenues in computational geometry, and computer vision to create powerful tools for $3 \mathrm{D}$ artists. We will discuss about some of these notable algorithms in this survey paper.

Sketch Based Modeling has undergone considerable amount of research over the past two decades with researchers investigating novel and innovative techniques at a rapid pace. So far researchers in the field of SBM have aimed their research on organic and inorganic modeling (architectural and mechanical) as stated by [Rivers et al. 2010]. Therefore, the research in SBM has been broadly divided into Organic Sketch Based Modeling and Inorganic Sketch Based Modeling. Inorganic $\mathrm{SBM}$ involves $\mathrm{CAD} / \mathrm{CAM}$ systems, architectural / building modeling systems and computer aided automobile design system. Notable examples of inorganic SBM systems are Google SketchUp [SketchUp], [Schmidt et al, 2009], and more recently [Rivers et al. 2010]. On the other hand organic sketch based modeling systems provide tools to create character models mainly using smooth feature curves, and suggestive contours [DeCarlo et al. 2003] etc. Organic modeling systems also provide tools for creating 3D models from simple primitives such as ellipsoids and using inflation techniques to inflate a closed $2 \mathrm{D}$ region / sketch (e.g. a circle, oval etc) such as Teddy [Igarashi et al. 2007], and FiberMesh [Nealen et al. 2007].

In order to effectively investigate and experiment new techniques in SBM, it is very important to possess a basic understanding of how traditional artists draw sketches of human characters, and how 3D artists model human like characters. Studying the process of sketching and drawing by artists can be found in a study by Cole et al [Cole et al, 2008]. Several excellent surveys are available on sketch based modeling techniques including [Olsen et al, 2009], and [Cook \& Arvin, 2009].

Sketch based modeling techniques are usually categorized into construction based and recognition based as indicated by Olsen et al (Olsen et al. 2009). Construction based techniques tend to generate a 3D model directly from 2D sketched feature curves while recognition based techniques uses 3D search engines to retrieve $3 \mathrm{D}$ models from the database which are similar to the $2 \mathrm{D}$ input sketches by applying shape descriptors to the input or matching feature curves to their counterparts in the 3D model database. Some notable example of 3D search engines are Princeton 3D search engine [Funkhouser et al, 2003], Konstanze University (CCCC) 
3D benchmark, McGill Shape Benchmark (MSB) [Zhang et al, 2005], National Taiwan University database (NTU) [Chen et al, 2003], and CEASAR human shape database [Allen et al, 2003]. A major problem faced when designing sketch based modeling techniques is the reconstruction of $3 \mathrm{D}$ objects directly from very limited information. Olsen et al [Olsen et al, 2009] have also concluded in their survey that a hybrid system that contains a substantial shape memory, robust creation rules, and perhaps even a capacity to learn new shapes, hold the most potential for approaching human-like sketch understanding.

Most artists require tools that could provide them the feeling of sketching on a canvas in a free hand manner. To address this fact, many SBM systems have been published and patented which give the users an experience of free hand sketching. In ILoveSketch [Bae et al. 2008], the authors have designed a curve sketching system that allows artists to draw strokes on a 3D canvas, and automatically approximates the strokes using NURBS. Several mouse inputs are mapped to navigation of the 3D canvas. Several gestures are provided for aligning the curves with other curves, scaling, rotation, and erasing curves.

\section{SBM Pipeline}

Generally speaking, SBM systems follow a systematic pipeline [Olsen et al, 2009], with each step employing several unique algorithms. The main steps in the pipeline are: 1) Sketch Acquisition, 2) Sketch Filtering, and 3) Sketch Interpretation.

2.1. Sketch acquisition: Sketch acquisition involves making choices about the medium and input devices for acquiring and drawing a sketch. Recent techniques have supported pen as input medium and drawing tablets as drawing canvas [Bae et al, 2008]. More haptic ways of 3D sketching have also emerged such as SPACESKETCH [Nam et al, 2012]. SPACESKETCH is a unique application that performs shape modeling by using 2 space wands in a $3 \mathrm{D}$ environment. By using a 3DTVdisplayas shown in Figure 1, sketching an object in a stereoscopic space is made possible.

2.2. Sketch filtering: Before a given sketch can undergo processing to be promoted to a $3 \mathrm{D}$ model, most state-ofthe-art techniques perform pre-processing on the input curves or strokes. This step is necessary to refine the input curves to be ready for processing. Denoising is an important class of algorithms for sketch refinement being employed heavily in SBM systems.

2.3. Sketch Interpretation: Sketch interpretation involves the transformation of 2D sketched contours into 3D surface mesh. This is the main component of an SBM system which includes several algorithms for generation of 3D geometry from input strokes.

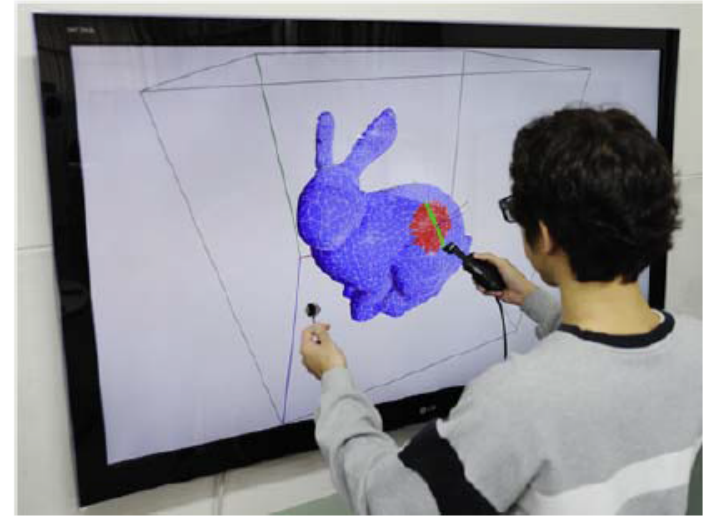

Fig 1: 3D Sketching with SPACESKETCH. (Reproduced from [Nam et al, 2012])

In this paper, we have categorized the techniques into the following major classes. 1) Single View systems, 2) Multi-View Systems, 3) Curve Networks Based Systems, 4) Data Driven Systems

\section{Single View Systems}

Single view SBM systems allow the artists to create models using just one sketch. These systems make use of algorithms to create surfaces which give an appearance of different parts of the human body, such as an ellipsoid which is an inflated silhouette of a 2D ellipse.

\subsection{Teddy: a sketching interface for $3 D$ freeform} design: Igarashi et al [Igarashi et al, 2007], pioneered the research in inflated geometrical surfaces to create simple organic toy like character models. Their interface is designed to quickly and easily model freeform surfaces for creating stuffed animals or other organic models. The system includes tools to add $3 \mathrm{D}$ geometry to the model when the user sketches the outline. The algorithm first finds the spine of the silhouette by using the chordal axes introduced in [Prasad 1997]. The system then wraps the spine with the polygonal mesh and then uses constraint Delaunay triangulation of the polygon. Pruning of insignificant branches is performed using [Prasad 1997]. The algorithm progresses through several refinement steps to obtain a smooth and symmetric 3D surface. Their system also provides tools for extruding and cutting of the mesh. Extrusion on the 3D surface is implemented using a sweeping algorithm. Some disadvantages of the Teddy system are: 1) it does not accepts complex and unexpected strokes such as Tjunction strokes and cusps. 2) The features provided are limited it was not suited to modeling complex and production ready models. In figure 2 , note that where the neck contour passes behind the chin, we see a $\mathrm{T}$ shape in the projected contour (called a T-junction), and the chin contour ends abruptly (called a cusp). T-junctions and cusps indicate the presence of a hidden contour; Williams [86] has proposed a method for using these to infer hidden contour lines in an image. 


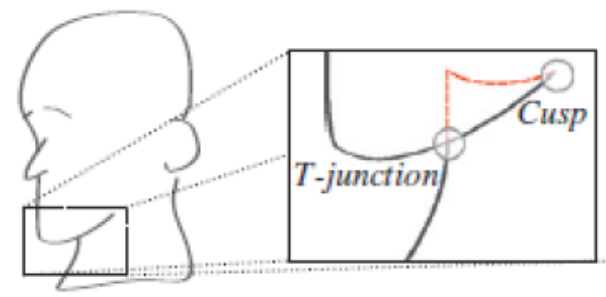

Fig 2: T-junction and cusp. (Reproduced from [Sketch-based modeling: a survey])

\subsection{SmoothSketch: 3D free-form shapes from complex} sketches: Several researchers have proposed improvements to the Teddy system. Karpenko \& Hughes [Karpenko \& Hughes, 2006] have addressed the problem of T-junctions and cusps by introducing an efficient algorithm extended from the work by William [Williams \& Allen, 1996] for inferring shapes with hidden contours, on figural completion of hidden contours containing T-junctions to contours containing cusps as well. It should be noted that SmoothSketch does not offers a complete SBM system; instead this work can be used as a component to be embedded in SBM systems. Williams' thesis included 3 steps to find the 3D surface to fit the given contours. These were 1) Drawing completion by inferring hidden contours, 2) Map the contours to the abstract topological surface and map the surface to $\mathbf{R}^{2}$, and 3) Lift this mapping to a smooth surface in $\mathbf{R}^{3}$, whose projection is the mapping to $\mathbf{R}^{2}$.

\subsection{FiberMesh: designing freeform surfaces with $3 D$} curves: Nealen et al have introduced a system called FiberMesh [Nealen et al, 2007] which is based on Teddy, but overcomes some of its limitations. Unlike Teddy, the user-drawn strokes stay on the model surface and serve as handles for controlling the geometry. The user can add, remove, and deform these control curves easily, as if working with a $2 \mathrm{D}$ line drawing. The curves can have arbitrary topology; they need not be connected to each other. For a given set of curves, the system automatically constructs a smooth surface embedding by applying functional optimization. The system also provides tools for model deformation such as adding ridges and creases to the 3D surface, and also cutting, extruding, pulling and tunnelling the geometry of the model using simple mouse movements. Figure 3 shows all the operations that FiberMesh offers to be performed on a mesh.

FiberMesh is a research bred SBM system that can be utilized for modeling organic models. An advantage of its interface is that the user does not need to worry about the topology of the curves. Traditional methods require the user to cover the entire surface with triangle or quad regions. In FiberMesh curves need not be connected to other curves and much fewer curves can represent simple geometry. It is also important that, instead of providing individual points as an interface, the interface treats curves as continuous entities. Its main disadvantage is that it is not suited for production ready models that can be modelled with professional modeling packages such Maya or ZBrush.

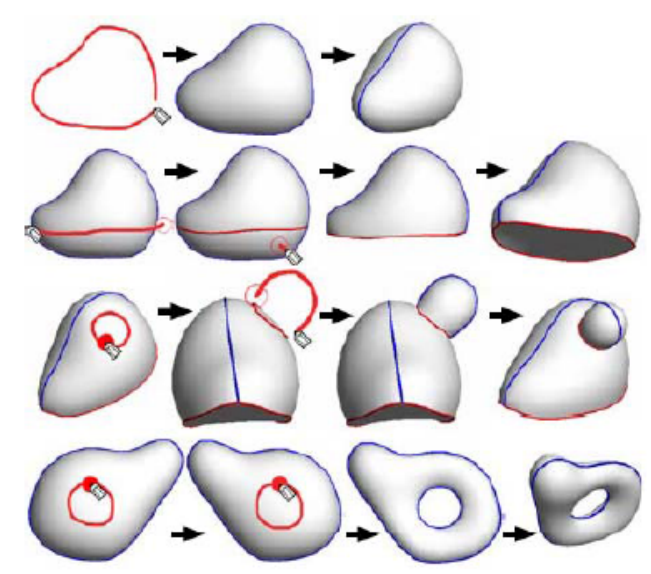

Fig 3: Sketching operations in FiberMesh (from top to bottom): creation, cut, extrusion and tunnel. (Reproduced from [Nealen et al, 2007])

3.4. Structured annotations for 2D-to-3D modeling: In [Gingold et al. 2009], Gingold et al have used generalized cylinder and ellipsoids for modeling of organic models directly from a single view model. The authors have used geometric primitives: Generalized Cylinder, and Ellipsoid to quickly model a simple lowpolygon model. Moreover the authors have provided annotations to make it easier for the authors to create plausible character with easy to use tools. The annotations - same-lengths and angles, alignment, mirror symmetry, and connection curves-allow the user to communicate higher level semantic information; through them their system builds a consistent model even in cases where the original image is inconsistent.. Figure 4 shows an overview of this approach. One disadvantage of this system is that it provides a limited set of tools to the artist for modeling and only provide two primitives (cylinder and ellipsoid). Most artists make use of more primitives to model a human character. One important primitive used by artists is the box primitive as demonstrated in tutorial video by the leading comic artist Stan Lee (www.youtube.com/watch?v=vhJ9CNU723A). In this tutorial, he tells us how easily one can decompose a human body into simple primitive geometric forms.

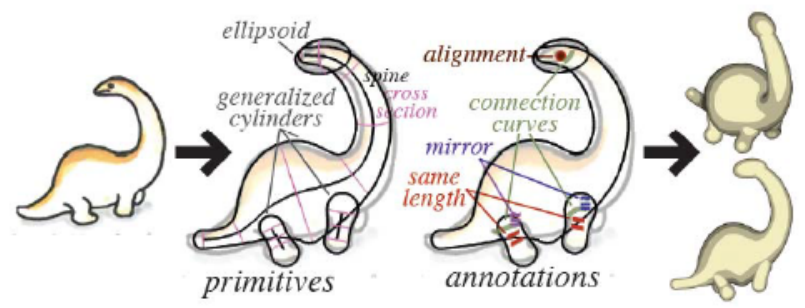

Fig 4: Modeling process of [Gingold et al. 2009]. The user places primitives and annotations on an image resulting in a 3D model. (Reproduced from [Gingold et al. 2009]) 


\subsection{Automatic single-view character model} reconstruction: Buchanan et al [Buchanan et al. 2013] have demonstrated a system which generates lowpolygon models directly from input character sketches without any interactivity by the artist. Their algorithm first computes a skeleton from the image and then draws cross section curves along the skeleton using a novel proposed heuristic. The overall benefit of the technique is to create a complete low-polygon base mesh model with the single click of a button, however since it doesn't provide enough interactivity to the user; this tool is not suitable for modeling high-resolution character models with well-defined feature curves. Their approach also demonstrates a shell-based meshing algorithm that allows for the ability to change the cross-sectional profile of the model based upon the type of character and even the type of limb in the model. Additionally, it contains a low complexity automatic skeletonization algorithm for raster images, with an optional user-controlled complexity parameter. The algorithm converts 2D outlines to 3D meshes using a heuristic that balances the skeletal relevancy of the mesh against reduced visual artefacts, using line style metrics to influence the $3 \mathrm{D}$ style of the generated mesh. By extracting a skeleton structure, approximating the $3 \mathrm{D}$ orientation and analysing line curvature properties, appropriate centrepoints can be found around which to create crosssectional slices and build the final triangle mesh. This technique can be useful for rapidly generating sub parts of the human character, such as limbs and torso as these parts are not very complicated anatomically. Figure 5 shows an overview of the process in [Buchanan et al. 2013].

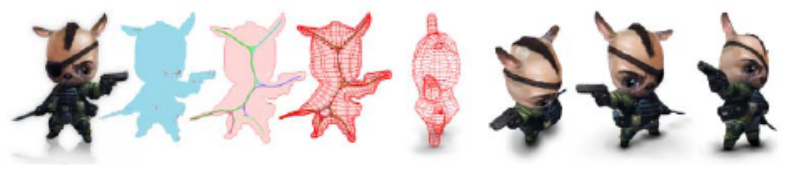

Fig 5: Overview of process in [Buchanan et al. 2013]. (Reproduced from [Buchanan et al. 2013)

3.6. Geosemantic Snapping for Sketch-Based Modeling: In [Shtof et al, 2013] Shtof et al have introduced a snapping technique which automatically reshapes and snaps simple $3 \mathrm{D}$ geometric primitives to 2D sketch primitives, then improves the model globally by inferring geosemantic constraints that link the different parts. The authors have used non-linear nonconvex optimization techniques (Augmented Lagrangian Method) to accomplish the task. The system requires the user to manual place the $3 \mathrm{D}$ primitives to their appropriate places as humans are better at this task than computers. The computer performs the tedious and precise alignments and snapping in real-time. The automatic identification of geosemantic relationships between the primitives such as co-planarity and continuity is an attractive feature of this system. The snapping behaviour of this system can be utilized to model several complex muscles of the human character which otherwise can be a tedious task. Figure 6 demonstrates an overview of [Shtof et al, 2013].
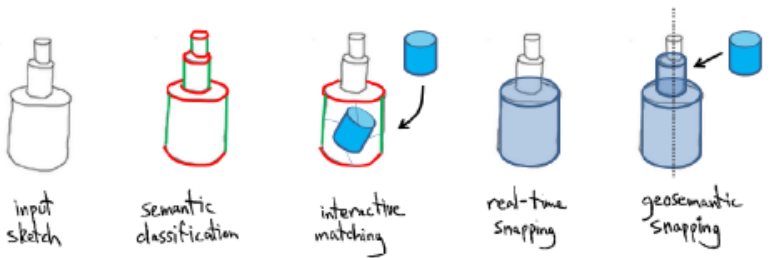

inpot
sketch

semantic
dassification

interactive
matching

real-time

geosemantic

Fig 6: Overview of the process in [Shtof et al, 2013]. (Reproduced from [Shtof et al, 2013])

Single-view sketch based modeling: Andre and Saito [Andre \& Suguru, 2011] have proposed a single view SBM system which is also based on the sweeping algorithm (generalized cylinders) but requires the user to draw two outlines (cross section of the shape and a closed curve perpendicular to the cross section) and an optional silhouette. In return the system sweeps the cross section along the silhouette outline curve. The attractive feature of this system is that the user draws the shape in the desired pose and uses a single view. This system is an important contribution towards SBM as it provides a very easy interface to the artist where the artist merely draws the $3 \mathrm{D}$ models as if they are drawing on a piece of paper, as their approach mimics the way most professional artists draw cartoonish characters. Unfortunately the models created from this method are rough and when modeling complex shapes made up of several sub-shapes, do not maintain smooth topology from one sub-shape to another in an organic manner, and therefore this system alone is not suitable for organic modeling of characters. Figure 7 demonstrates an overview of the process of this approach.
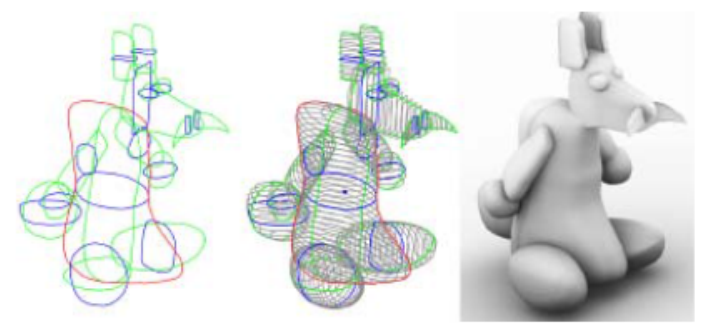

Fig 7: Overview of process in [Andre \& Suguru, 2011]. (Reproduced from [Andre \& Suguru, 2011])

3.7. 3-Sweep: Extracting Editable Objects from a Single Photo: In [Chen et al, 2013], the authors have demonstrated a 3D model reconstruction technique from single photos, by automatically fitting the boundaries of generalized cylinder to the boundaries of the subject in the image. It requires the users to first draw a profile curve of the generalized cylinder, which are essentially an ellipse in 3D space. The user then drags the mouse over the 2D shape and the system renders a generalized cylinder along the path swept by the mouse/pen and snaps it to the outline of the $2 \mathrm{D}$ shape. The algorithm contains computing the $2 \mathrm{D}$ shape, its projections and its 
relations to other shapes in the image using energy minimization methods, thus simulating the cognitive ability of humans. This system makes the daunting task of extracting objects simple, and snapping the $3 \mathrm{D}$ object to the $2 \mathrm{D}$ image outline. Thus this system comes out to be an intelligent and useful system for our research topic of sketch based modeling of realistic character models. This system is focused towards modeling of objects made up of pipes and cylinder such as vases, telescopes, binoculars, pots and other similar solid body mechanical object, and doesn't suit organic modeling. This is an important limitation of this approach.

\section{Multi-view systems}

Several multi view 3D reconstruction techniques are available which transform a set of images taken from different views of a model into a complete 3D model such as (Lin et al. 2010), and Autodesk 123D catch (www.123dapp.com/catch).

4.1. $3 D$ modeling with silhouettes: Rivers et al [Rivers et al, 2010] have proposed a new and simple algorithm towards computing the silhouette cylinders to compute the 3D Constructive Solid Geometry (CSG) by leveraging the special properties of silhouette cylinders. The main idea behind their approach is that the silhouettes of the sub-parts of a 2D drawing from two different views (front and side) can give enough information to create a 3D model. In their interface, a user specifies the silhouettes of a part from front, side, or top views. Once two or more silhouettes have been specified its 3D shape is automatically constructed. Although each part is axis-aligned, parts can be rotated in $3 \mathrm{D}$ relative to each other, such that the model as a whole need not be axis-aligned. The system targets the modeling of man-made objects, as they typically can be decomposed into axis-aligned subparts. Organic models are not well suited to this approach. The algorithm combines the parts of the 3D shape using Boolean operations. Figure 8 shows the overview of the process in [Rivers et al, 2010].

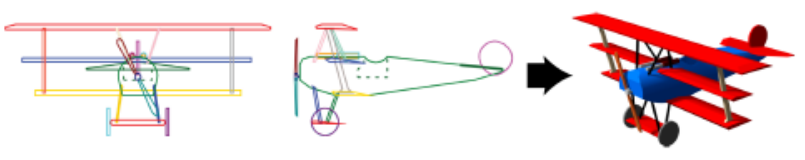

Fig 8: Overview of the process in [Rivers et al, 2010]. (Reproduced from [Rivers et al, 2010])

The algorithm implemented by the authors proceeds in the following steps: 1) Find the intersection of each silhouette cylinder $c$ with the plane. 2) Label these intersection polygons $i_{c}$. 3) For each silhouette, also find the intersection of the surface of the silhouette cylinder with the plane (as opposed to the interior), labelled $s_{c}$ which is a subset of $i_{c}$. 4) Apply 2D Boolean operations to the intersection and surface polygons corresponding to a set of silhouette cylinders to compute $i$ and $c$ for the solid resulting from a Boolean operation applied to the corresponding silhouette cylinders. In general, the authors have shown that Boolean operations can be computed for any two solids for which $\mathrm{i}$ and $\mathrm{s}$, the interior and surface polygons, are known for every plane, to yield the $i$ and $s$ of the resulting solid for each plane, allowing further operations to be applied to the derived solid. For smoothing of the 3D models, the authors have adopted the smoothing algorithm from FiberMesh. This smoothing operation generates a surface that approximates the surface minimizing the variation of Laplacian magnitude
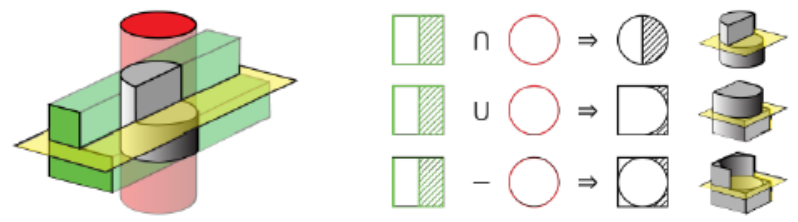

Fig 9: Modeling using Boolean operations. (Reproduced from [Rivers et al, 2010])

\section{Curve Networks Based Systems}

Sketch based modeling systems have benefited substantially with the $3 \mathrm{D}$ space available to the artists to draw strokes. A curve network as the name suggests is a network of strokes that the artist draws on a 2D canvas and they are transformed into $3 \mathrm{D}$ curves by underlying algorithms. In this section we will look into two systems based heavily on curve networks drawn in 3D space.

5.1. ILoveSketch: Bae et al [Bae et al, 2008] have developed a robust and feature rich system for $3 \mathrm{D}$ sketching. The salient features behind the design of this system are:

- Visually smooth complex curves for design exploration

- Minimal interruption to sketching by GUI and gestures

- Minimal set of gestures with intrinsic affordances

- Immediate and easy access to $2 \mathrm{D} / 3 \mathrm{D}$ navigation

- Dynamic information display to assist 2D/3D sketching

- Focus on geometric objects rather than UI components

The basic "feel" of the system borrows from that of a physical paper sketchbook. This system provides designers with a virtual sketchbook with tools for smooth navigation of multiple canvases with interactions such as: tearing, peeling, panning, zooming, and rotation. The system also support automatic dynamic rotation of the virtual sketchbook based on the users' input strokes to make further multi-stroke sketching biomechanically comfortable. However according to the case study 
mentioned in the paper, one artist did not find the automatic dynamic rotation of view convenient and rather distracting. Figure 9 shows some models created using [Bae et al, 2008].

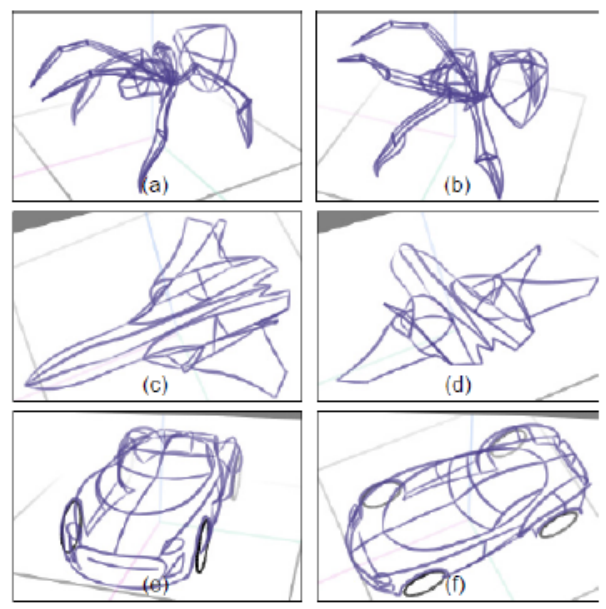

Fig 9: Models created using [Bae et al, 2008]. (Reproduced from [Bae et al, 2008])

The authors have provided five different 3D curve sketching methods along with the notion of sketchability - a view dependent scalar measure that helps determine how good a given viewing angle is for a given $3 \mathrm{D}$ curve sketching method. Sketchability-based automatic 3D rotation increases a designer's throughput, by reducing the need for explicit 3D navigation to find a suitable view in which to sketch. A minimal gesture set is provided for command input, and audio feedback is used to support gesture confirmation. As a whole, these methods result in a coherent 3D curve sketching workflow that does not rely on menus, icons, or tool palettes that could clutter the screen. Thus, as argued by the authors the user's focus of attention can stay on the artwork at all times. One limitation of this system is that it is not suitable for modeling organic character models as most of the models demonstrated using this system are mechanical in nature such as aircrafts, cars, and spaceships etc.

5.2. JustDrawIt: Similar in spirit with the ILoveSketch [Bae et al, 2008] system, Grimm \& Joshi [Grimm \& Joshi, 2012] have proposed 'JustDrawIt'. It is a 3D curve sketching system based on existing and novel techniques. JustDrawIt provides an interface to the artists to draw 2D strokes and then the system converts the strokes into 3D curves. The system support "snapping" together curve networks and specifying normal in order to create consistent curves from which surface models can be generated. Figure 10 shows the modeling process of JustDrawIt.

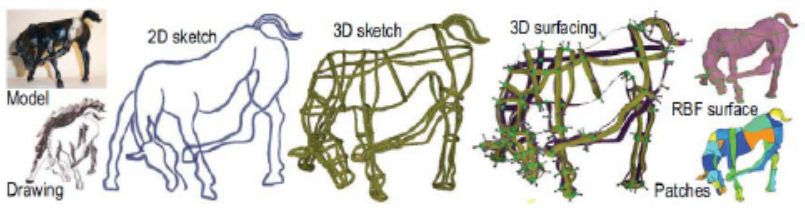

Fig 10: Modeling process of [Grimm \& Joshi, 2012]. (Reproduced from [Grimm \& Joshi, 2012])

At the core of this system lies the stroke inference engine, which infers the stroke, refines it and transform into curves. The system requires the users to roughly input some strokes from the input device such as a pen. Artists when drawing usually draw in a discontinuous fashion. Back and forth, over-sketched, multi-stroking and disjoint strokes are common in traditional drawing practices. The system tolerates these rough user inputs of strokes and converts the strokes into curves. The user is able to perform different manipulation operations on the curves such as (dragging, scaling, rotating, smoothing, erasing some or all) by clicking on a curve, and selecting the desired option from the curve menu.
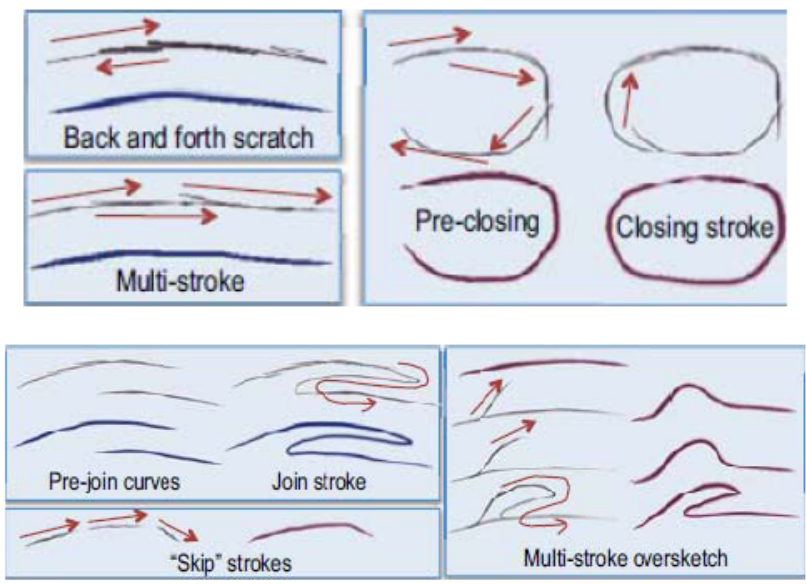

Fig 11: Stroke joined into curves and stroke refinement [Grimm \& Joshi, 2012]. (Reproduced from [Grimm \& Joshi, 2012])

After the user has drawn a few curves, he/she can proceed towards adding depth (in 3D) to the curve by dragging the curve in the view direction (z-axis).

In contrast with ILoveSketch, this system does not use epipolar constraints [KHR04, BBS08] to specify depth values along non-planar curves. Instead, but treats the problem as one of oversketching [CMZ*99]. It is very difficult for a user to envision what a curve would look like from two different views, so instead this system always create a $3 \mathrm{D}$ curve. The user can then change the view and oversketch or continue that curve from the new view. The authors have used a novel depth interpolation and extrapolation technique to make the new stroke consistent (in depth) with the existing curve. For 3D surface creation the system provides visualization and interface support for automatically and semiautomatically snapping curves together and orienting 
them. In particular, a novel ribbon rendering method is used, which makes visualizing and editing the curve orientation (which direction is "out") easier.

JustDrawIt benefits from its 2D drawing interface which is easy and intuitive for the artists to learn, however its main disadvantage is in its $3 \mathrm{D}$ curve drawing aspect, which has a steep learning curve. Also the artists have also stated that they have not performed a formal user study and only tested the system with four experienced users and allowed them to experiment with it.

\section{Data Driven Systems}

Researchers have proposed several novel morphing techniques and found them to be attractive techniques towards generating 3D models from several posed models in a database, and 2D images. These systems are essentially $3 \mathrm{D}$ search engines making heavy use of $3 \mathrm{D}$ and $2 \mathrm{D}$ shape databases to guide the user towards modeling plausible 3D models. These systems employ $2 \mathrm{D}$ and $3 \mathrm{D}$ shape descriptors to retrieve parts of the models from databases as well as complete models. Several good surveys exist that give an excellent overview of state-of-art shape descriptors such as [2], [Zhang \& Lu, 2004], [Zhang et al, 2007], [Yang et al, 2007], and [Kazmi et al, 2013]. In this section we will discuss a recent and powerful SBM systems based on 3D search engines.

6.1. $\boldsymbol{B o F + G A L I F}$ : Eitz et al [Eitz et al, 2012] have developed a $3 \mathrm{D}$ search engine which utilizes existing and novel algorithms in its pipeline. This system accepts a rough user sketch as input and matches the sketch with several sketched renderings of the $3 \mathrm{D}$ model present in the database. A $3 \mathrm{D}$ model in the database is first subject to different camera view captures. Then each view is rendered as a line drawing using Suggestive Contours algorithm introduced in [DeCarlo et al, 2003]. Then a shape descriptor is computed using all line drawings using Gabor Filters algorithm. A visual vocabulary is developed from all the models in the databases using the Bag-of-Features approach. The input sketch is also subject of Gabor Filters and then the feature vector is matched with the visual vocabulary to find the closest matches.

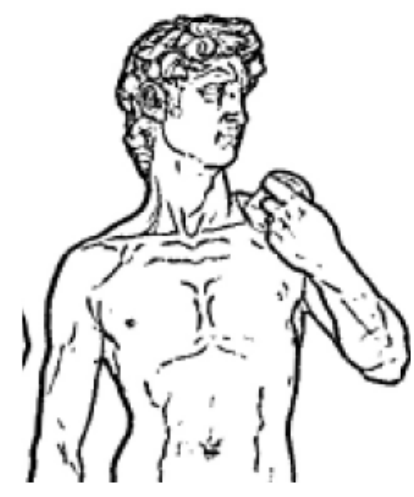

Fig 12: Suggestive contours algorithm applied to the David statue 3D model [DeCarlo et al, 2003]. (Reproduced from [DeCarlo et al, 2003])

6.3. A Data-Driven Approach to Realistic Shape Morphing: This is a data-driven approach that aims at computing morphed models by using models already present in the database. The models in the database are clustered to form local shape spaces. To find the closeness between the pairs of models in the clusters, a simple distance metric is used. The morphing problem is then solved by solving a global optimization problem of finding a minimal distance path within the local shape spaces. For producing the final models, an extended as rigid as possible interpolation is used. The authors have casted the morphing problem as a global optimization problem of finding a shortest path from the source to the target with intermediate models from the local shape spaces using the distance measure.

\section{Morphing based systems}

7.1. Modeling from Contour Drawings: In this approach by [Kraevoy et al, 2009], a contour drawing is used as an input drawing and a correspondence is found between the given drawing and a template model to produce the final model. To produce the final morphed model, an optimization problem is solved using Hidden Markov Model to find the optimal correspondence between a sequence of $2 \mathrm{D}$ stroke points and $3 \mathrm{D}$ template vertices. The second contribution of the authors is that they have develop an iterative correspond and deform framework which is key to making this sketch based modeling work. The input drawing is represented as a sequence of points with associated outward pointed normals. The task of finding a correspondence between a sequence of $3 \mathrm{D}$ vertices for every sketched contour stroke, i.e. finding a continuous sequence of vertices that best match with the continuous sketched contours. This calls for solving an optimization problem because the aim here is to minimize the transition costs when the distances travelled along the drawn contour and between $3 \mathrm{D}$ vertices are equal. To solve this optimization problem, the authors have used the Hidden Markov Model and Viterbi algorithm (dynamic programming solution). 
For the HMM, the points are treated as a set of observation states, while the $3 \mathrm{D}$ vertices to be inferred are treated as a set of hidden states. HMM requires emission probabilities and transition probabilities. These are solved using the formulas given in the paper. The deformation of the template model to match the sketched contours is performed iteratively using mean value encoding.

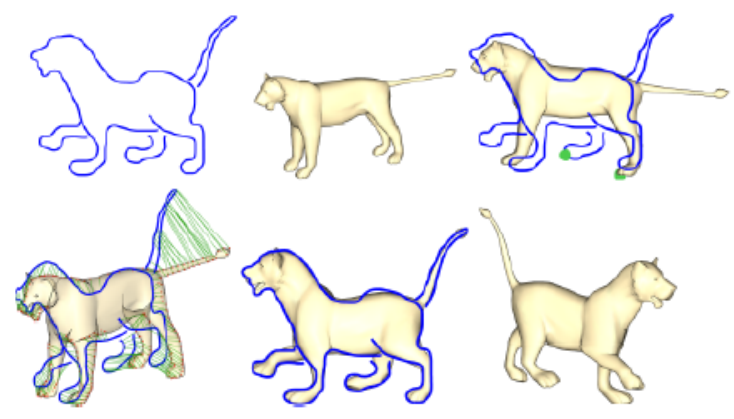

Figure 13: Overview of morphing process of [Kraevoy et al, 2009]. (Reproduced from [Kraevoy et al, 2009])

One of the limitations with the proposed technique is the use of HMM technique to solve the optimization problem, which is slow to compute and can take up to 1 to 2 minutes to find the optimal correspondence.

\subsection{What Shape are Dolphins? Building Morphable models from 2D images: In this approach from Cashman et al [Cashman et al, 2013], the authors have used several images and a template model as inputs, to compute a morphable model. Given a set of images, the approach first extracts the silhouette of the image and the silhouette is sampled with discrete points and associate normal with these normal. Each image is also accompanied with user specified point constraints, which help to direct the optimization into the correct energy well. Each image is also associated with camera projection parameters to represent each image to lie in the xy plane facing the z-axis. With this setup, the authors have proposed an optimization algorithm, and the formulation of an objective function / energy function. The optimization algorithms match the image silhouette with the template model.}

\subsection{Human shape correspondence with automatically} predicted: landmarks In (Wuhrer et al. 2012), the authors have proposed a new approach for finding the correspondence between two 3D models in a database by automatically predicting the landmarks. The approach is composed of 3 main steps 1) Predicting landmarks, 2) Finding point-to-point correspondence, and 3) Evaluating the accuracy of the correspondence between the $3 \mathrm{D}$ models. At the core of this approach is the use a human body database CEASAR. This database is composed of thousands of scanned high resolution and realistic human models. For the prediction of landmarks, the authors have used a modified version of the algorithm proposed by Azouz et al in (Azouz et al. 2006). This algorithm identifies 73 landmarks on the human body by learning from a pairwise Markov Network. The actual correspondence between a template model and a scanned model is found by using a 2 step alignment process. Firstly the authors have utilized Radial Basis Functions (RBF) and secondly their approach deforms the template model using a non-linear optimization algorithm based on Broyden-FletcherGoldfarb-Shanno quasi-Newton approach (Liu \& Nocedal. 1989) to solve the optimization problem. The final step of the approach is 'Evaluation', in which the accuracy of the proposed approach is conducted over a large database of 500 human body scans. The accuracy is found using 3 measures: compactness, generalization, and specificity. The authors used a modified version of the landmarks prediction algorithm by compromising some amount of accuracy but gaining some amount of speed in computation. Loss of accuracy is one disadvantage the authors have mentioned in paper. One of the advantages of Radial Basis Function is that it is easy to compute and implement as it is a Neural Network that can be solved using simple linear algebra computations.

\section{Conclusion}

$3 \mathrm{D}$ modeling has a very long way to cover to match the convenience of drawing on paper and expressing imagination on paper. Interpreting different contour lines in a sketch by a machine is one of toughest problems in SBM. Same can be said for generating realistic 3D characters from 2D sketches. Curve network system can be a promising future for SBM as they provide a paradigm and interface for $3 \mathrm{D}$ sketching, which in nature shares a similar experience with 2D sketching on paper.

In this survey paper we investigated state of the art techniques in Sketch Based Modeling and pointed out their advantages and disadvantages and also classified these techniques into several categories.

\section{Acknowledgement}

This research is supported by the grant of 2013 UK Royal Society International Exchanges Scheme (Grant no. IE131367).

\section{References}

[Maya] Autodesk Inc., Maya (www.autodesk.com/maya)

[Zbrush] Pixologic Inc., Zbrush (www.pixologic.com)

[Mudbox] Autodesk Inc., Mudbox (www.autodesk.com/ mudbox)

[SketchUp] Google Inc., SketchUp (www.sketchup.com)

[Canny 1986] Canny, J. (1986). A computational approach to edge detection. Pattern Analysis and Machine Intelligence, IEEE Transactions on, (6), 679-698.

[Schmidt et al, 2009] Schmidt, Ryan, Azam Khan, Karan 
Singh, and Gord Kurtenbach. "Analytic drawing of 3D scaffolds." In ACM Transactions on Graphics (TOG), vol. 28, no. 5, p. 149. ACM, 2009.

[Rivers et al, 2010] Rivers, Alec, Frédo Durand, and Takeo Igarashi. 3D modeling with silhouettes. Vol. 29, no. 4. ACM, 2010.

[Igarashi et al, 2007] Igarashi, Takeo, Satoshi Matsuoka, and Hidehiko Tanaka. "Teddy: a sketching interface for 3D freeform design." In ACM SIGGRAPH 2007 courses, p. 21. ACM, 2007.

[Nealen et al, 2007] Nealen, Andrew, Takeo Igarashi, Olga Sorkine, and Marc Alexa. "FiberMesh: designing freeform surfaces with 3D curves." ACM Transactions on Graphics (TOG) 26, no. 3 (2007): 41 .

[Gingold et al, 2009] Gingold, Yotam, Takeo Igarashi, and Denis Zorin. "Structured annotations for 2D-to-3D modeling." In ACM Transactions on Graphics (TOG), vol. 28, no. 5, p. 148. ACM, 2009.

[Buchanan et al, 2013] Buchanan, Philip, R. Mukundan, and Michael Doggett. "Automatic single-view character model reconstruction." In Proceedings of the International Symposium on Sketch-Based Interfaces and Modeling, pp. 5-14. ACM, 2013.

[Prasad 1997] L. Prasad. Morphological analysis of shapes. CNLS Newsletter, 139: 1-18, July 1997.

[Karpenko \& Hughes, 2006] Karpenko, Olga A., and John F. Hughes. "SmoothSketch: 3D free-form shapes from complex sketches." In ACM Transactions on Graphics (TOG), vol. 25, no. 3, pp. 589-598. ACM, 2006.

[Grimm \& Joshi, 2012] Grimm, Cindy, and Pushkar Joshi. "Just Draw it! a 3D Sketching System." In 9th Eurographics symposium on sketch-based interfaces and modeling, pp. 1-14. 2012.

[Bae et al, 2008] Bae, Seok-Hyung, Ravin Balakrishnan, and Karan Singh. "ILoveSketch: as-natural-as-possible sketching system for creating $3 d$ curve models." In Proceedings of the 21st annual ACM symposium on User interface software and technology, pp. 151-160. ACM, 2008.

[Olsen et al, 2009] Olsen, Luke, Faramarz F. Samavati, Mario Costa Sousa, and Joaquim A. Jorge. "Sketch-based modeling: A survey." Computers \& Graphics 33, no. 1 (2009): 85-103.

[Nam et al, 2012] Nam, Sanghun, and Youngho Chai. "SPACESKETCH: Shape modeling with $3 \mathrm{D}$ meshes and control curves in stereoscopic environments." Computers \& Graphics 36, no. 5 (2012): 526-533.

[Zhang \& Lu, 2004] D. Zhang and G. Lu, "Review of shape representation and description techniques," Pattern Recognition, vol. 37, no. 1, pp. 1-19, Jan. 2004.

[Zhang et al, 2007] L. Zhang, M. Fonseca, and A. Ferreira, "Survey on 3D shape descriptors," 2007.

[Yang et al, 2007] Y. Yang, H. Lin, and Y. Zhang, "ContentBased 3-D Model Retrieval: A Survey," IEEE Transactions on Systems, Man and Cybernetics, Part C (Applications and
Reviews), vol. 37, no. 6, pp. 1081-1098, Nov. 2007.

[Kazmi et al, 2013] Kazmi, Ismail Khalid, Lihua You, and Jian Jun Zhang. "A Survey of 2D and 3D Shape Descriptors." In Computer Graphics, Imaging and Visualization (CGIV), 2013 10th International Conference, pp. 1-10. IEEE, 2013.

[Eitz et al, 2012] Eitz, Mathias, Ronald Richter, Tamy Boubekeur, Kristian Hildebrand, and Marc Alexa. "Sketchbased shape retrieval." ACM Trans. Graph. 31, no. 4 (2012): 31.

[DeCarlo et al, 2003] DeCarlo, Doug, Adam Finkelstein, Szymon Rusinkiewicz, and Anthony Santella. "Suggestive contours for conveying shape." In ACM Transactions on Graphics (TOG), vol. 22, no. 3, pp. 848-855. ACM, 2003.

[Kraevoy et al, 2009] Kraevoy, Vladislav, Alla Sheffer, and Michiel van de Panne. "Modeling from contour drawings." In Proceedings of the 6th Eurographics Symposium on SketchBased interfaces and Modeling, pp. 37-44. ACM, 2009.

[Cashman et al, 2013] Cashman, Thomas J., and Andrew W. Fitzgibbon. "What shape are dolphins? Building 3D morphable models from 2D images." Pattern Analysis and Machine Intelligence, IEEE Transactions on 35, no. 1 (2013): 232-244.

[Cole et al, 2008] Cole, Forrester, Aleksey Golovinskiy, Alex Limpaecher, Heather Stoddart Barros, Adam Finkelstein, Thomas Funkhouser, and Szymon Rusinkiewicz. "Where do people draw lines?." In ACM Transactions on Graphics (TOG), vol. 27 , no. 3 , p. 88 . ACM, 2008.

[Cook \& Arvin, 2009] Cook, Matthew T., and Arvin Agah. "A survey of sketch-based 3-D modeling techniques." Interacting with Computers 21, no. 3 (2009): 201-211.

[Funkhouser et al, 2003] Funkhouser, Thomas, Patrick Min, Michael Kazhdan, Joyce Chen, Alex Halderman, David Dobkin, and David Jacobs. "A search engine for 3D models." ACM Transactions on Graphics (TOG) 22, no. 1 (2003): 83105 .

[Allen et al, 2003] Allen, Brett, Brian Curless, and Zoran Popović. "The space of human body shapes: reconstruction and parameterization from range scans." In ACM Transactions on Graphics (TOG), vol. 22, no. 3, pp. 587-594. ACM, 2003.

[Chen et al, 2013] Chen, Tao, Zhe Zhu, Ariel Shamir, Shi-Min $\mathrm{Hu}$, and Daniel Cohen-Or. "3-Sweep: extracting editable objects from a single photo." ACM Transactions on Graphics (TOG) 32, no. 6 (2013): 195 .

[Wuhrer et al. 2012] Wuhrer, Stefanie, Pengcheng Xi, and Chang Shu. "Human shape correspondence with automatically predicted landmarks." Machine Vision and Applications 23, no. 4 (2012): 821-830.

[Shtof et al, 2013] Shtof, Alex, Alexander Agathos, Yotam Gingold, Ariel Shamir, and Daniel Cohen-Or. "Geosemantic Snapping for Sketch-Based Modeling." In Computer Graphics Forum, vol. 32, no. 2pt2, pp. 245-253. Blackwell Publishing Ltd, 2013.

[Andre \& Suguru, 2011] Andre, Alexis, and Suguru Saito. "Single-view sketch based modeling." In Proceedings of the Eighth Eurographics Symposium on Sketch-Based Interfaces 
and Modeling, pp. 133-140. ACM, 2011.

[Zhang et al, 2005] Zhang, Juan, K. Siddiqi, D. Macrini, A. Shokoufandeh, and S. Dickinson. "Retrieving articulated 3-d models using medial surfaces and their graph spectra," In Energy minimization methods in computer vision and pattern recognition, pp. 285-300. Springer Berlin Heidelberg, 2005.

[Chen et al, 2003] D.-Y. Chen, X.-P. Tian, Y.-T. Shen, and M. Ouhyoung, "On Visual Similarity Based 3D Model Retrieval," Computer Graphics Forum, vol. 22, no. 3, pp. 223232, Sep. 2003

[Williams \& Allen, 1996] Williams, Lance R., and Allen R. Hanson. "Perceptual completion of occluded surfaces." Computer Vision and Image Understanding 64, no. 1 (1996): 120. 